\title{
Revisão da Dimensão Isolamento do Inventário Dimensional Clínico da Personalidade
}

\author{
Lucas de Francisco Carvalho ${ }^{1}$ \\ Wellington Arruda \\ Universidade São Francisco, Itatiba, SP, Brasil
}

\begin{abstract}
Resumo
O presente estudo teve como objetivo revisar a dimensão Isolamento do Inventário Dimensional Clínico da Personalidade (IDCP). Para tanto, o método foi dividido em duas etapas, sendo a primeira voltada para elaboração de novos itens com base na literatura, e a segunda visou a verificação das propriedades psicométricas do novo conjunto de itens. O IDCP em conjunto ao Inventário de Personalidade NEO Revisado (NEO-PI-R) e o Personality Inventory for DSM-5 (PID-5) foram aplicados em uma amostra de 213 sujeitos, entre 18 e 69 anos $(M=25,56 ; D P=8,70)$, e maioria do sexo feminino $(74,3 \%)$. A primeira etapa resultou em uma versão de 39 itens, dos quais 28 eram novos. A segunda, resultou em uma dimensão composta por 18 itens, em quatro fatores interpretáveis de acordo com as análises de estrutura interna, sendo, Individualismo, Isolamento Social, Evitação de Intimidade e Apatia Emocional, com coeficientes de consistência interna de 0,88 para o total, e superior a 0,75 para os fatores. A correlação da dimensão com o NEO-PI-R e o PID-5 revelou magnitudes coerentes e esperadas. Os dados demonstram a adequação da nova dimensão Isolamento do IDCP.
\end{abstract}

Palavras-chave: Avaliação psicológica, traços de personalidade, psicometria, transtornos da personalidade, DSM-5.

\section{Review of the Isolation Dimension of the Dimensional Clinical Personality Inventory}

\begin{abstract}
The present study aimed to review the Isolation dimension of the Dimensional Clinical Personality Inventory (DCPI). The method was divided in two steps, the first focused on developing new items based on the literature, and the second for testing the psychometric properties of the new items dataset. The DCPI plus the Revised NEO Personality Inventory (NEO-PI-R) and the Personality Inventory for DSM-5 (PID-5) were applied in a sample of 213 subjects aging between 18 and 69 years $(M=25.56 ; S D$ $=8.70)$, with predominance of women $(74.3 \%)$ The first step resulted in 39 items, of which 28 were new. The second stage results on a composite of 18 items, in four interpretable factors based on the internal structure analysis, Individualism, Social Isolation, Intimacy Avoidance and Emotional Apathy with internal consistency coefficients of .88 for total, and higher then .75 for each factor. The correlations of the scale with NEO-PI-R and PID-5 revealed consistent and expected effect sizes. The data reveal the adequacy of the new Isolation dimension of DCPI.
\end{abstract}

Keywords: Psychological assessment, personality traits, psychometrics, personality disorders, DSM-5.

Endereço para correspondência: Universidade São Francisco, Rua Alexandre Rodrigues Barbosa, 45, Itatiba, SP, Brasil 13251-900. Fone: (11) 4534-8053. E-mail: lucas@labape.com.br e psbwell@yahoo.com.br Financiamento: Fundação de Amparo à Pesquisa do Estado de São Paulo (FAPESP). 


\section{Revisión de la Dimensión Aislamiento del Inventario Dimensional Clínico de la Personalidad}

\section{Resumen}

El presente estudio tuvo como objetivo revisar la dimensión Aislamiento del Inventario Dimensional Clínico de la Personalidad (IDCP). Para eso, el método se divide en dos etapas, la primera que se centra en el desarrollo de nuevos elementos en función de la literatura, y el segundo tenía como objetivo verificar las propiedades psicométricas de los ítems nuevos. El IDCP fue aplicado junto al Inventario de Personalidad NEO Revisado (NEO-PI-R) y el Personality Inventory for DSM-5 (PID-5), en una muestra de 213 sujetos con edades entre 18 y 69 años $(M=25.56 ; S D=8.70)$, siendo la mayoría mujeres (74.3\%). La primer etapa produjo una versión de 39 ítems, de los cuales 28 eran nuevos. El segundo dio lugar a una mezcla compuesta de 18 ítems en cuatro factores interpretables según el análisis de la estructura interna, siendo ellos: individualismo, aislamiento social, evitación de la intimidad y apatía emocional, con coeficientes de consistencia interna de .88 para la dimensión total, y más de .75 para los factores. La correlación con el NEO-PI-R y el PID-5 mostró magnitudes coherentes y esperadas. Los datos demuestran la adecuación de la nueva dimensión de aislamiento de IDCP.

Palabras clave: Evaluación psicológica, rasgos de personalidad, psicometría, transtornos de la personalidad, DSM-5.

O modelo categórico utilizado para a classificação e diagnóstico dos transtornos de personalidade tem sido alvo de diversas críticas (Skodol et al., 2011; Zimmerman, 2011). A falta de critérios válidos para a definição dos perfis clínicos, o excesso de comorbidade nos diagnósticos realizados tendo como base esse modelo, a arbitrariedade evidente nas distinções entre o saudável e o patológico e a discordância em relação aos critérios constituintes de cada transtorno, destacam-se como questões que apresentam os limites da proposta categórica. Por isso, vem ganhando força na literatura a proposta dimensional para avaliação de características patológicas da personalidade e diagnóstico dos transtornos da personalidade, que basicamente propõe um continuum entre as características saudáveis e patológicas da personalidade, de modo que todos os indivíduos possuem todas as características, contudo, em diferentes níveis ou severidade (Kendler, Kupfer, Narrow, Phillips, \& Fawcett, 2009; Widiger \& Lowe, 2008).

Foi desenvolvido no Brasil o Inventário Dimensional Clínico da Personalidade (IDCP; Carvalho, 2011; Carvalho \& Primi, in press), composto por 12 dimensões que avaliam características patológicas da personalidade. Os estu- dos realizados com o instrumento demonstram sua adequação psicométrica, contudo, pesquisas aprimorando as dimensões do IDCP vem sendo realizadas no sentido de acumular evidências de validade para o instrumento e refinamento de sua capacidade avaliativa. O presente estudo enquadra-se nesse grupo de estudos, na busca por revisar uma das dimensões do IDCP que visam avaliar características patológicas da personalidade, a dimensão Isolamento.

As características patológicas da personalidade podem se manifestar nos indivíduos de maneira a representar transtornos da personalidade. Esses transtornos podem ser caracterizados por três atributos amplos (Millon, 2011), a inflexibilidade adaptativa, o circulo vicioso e a estabilidade tênue. A inflexibilidade adaptativa relaciona-se a falta de habilidades e estratégias no enfrentamento das dificuldades assim como na busca da realização de objetivos; o circulo vicioso trata-se de um esquema criado e mantido pelo indivíduo, de retroalimentação das dificuldades e prejuízos existentes; a estabilidade tênue refere-se à baixa resiliência do sujeito frente às situações estressoras ambientais. Além disso, esses transtornos podem ser identificados pela presença estável de prejuízos nas relações inter- 
pessoais e na percepção do eu/self (Skodol et al., 2011).

Em uma perspectiva internacional, são observados diversos instrumentos de autorrelato para avaliação de características patológicas da personalidade (Millon, Millon, Meagher, Grossman, \& Ramanath, 2004), as quais eventualmente podem configurar transtornos da personalidade. Exemplo disso é o Minnesota Multiphasic Personality Inventory 2 (MMPI-2), o Millon Clinical Multiaxial Inventory III e o Personality Inventory for DSM-5 (PID-5), cuja natureza é de autorrelato em sua maioria. Contudo, a maior parte desses testes não foi adaptada para o Brasil ou não possui estudos psicométricos no país. Nesse sentido, publicações nacionais apontaram a escassez de instrumentos nessa área no Brasil (Carvalho, Bartholomeu, \& Silva, 2010).

Levando em conta essa lacuna no país, Carvalho e Primi (in press) desenvolveram o Inventário Dimensional Clínico da Personalidade (IDCP), partindo dos critérios diagnósticos do Manual Estatístico e Diagnóstico de Transtornos Mentais IV-TR (DSM-IV-TR; American Psychiatric Association [APA], 2003) e da teoria de Millon (Millon, Grossman, \& Tringone, 2010; Millon et al., 2004). Trata-se de um instrumento de autorrelato constituído por 163 itens que devem ser respondidos em uma escala tipo Likert de 4 pontos. Os itens do IDCP estão agrupados em doze dimensões (Dependência, Agressividade, Instabilidade de Humor, Excentricidade, Necessidade de Atenção, Desconfiança, Grandiosidade, Isolamento, Evitação à Críticas, Autossacrifício, Conscienciosidade e Impulsividade) que apresentaram propriedades psicométricas adequadas no que se refere aos índices de fidedignidade com base na consistência interna ( $\alpha$ de Cronbach superior a 0,70 para quase todas as dimensões, excluindo-se Conscienciosidade que obteve 0,69 ) e evidências de validade com base na estrutura interna (análise fatorial exploratória e calibração com índices de ajuste pelo rating scale model) e com base em variáveis externas, quais sejam, diagnóstico psiquiátrico e NEO-PI-R (Carvalho, 2011; Carvalho \& Primi, in press; Carvalho, Primi, \& Stone, 2014a).
Apesar das adequações psicométricas do IDCP, de acordo com os apontamentos levantados por Carvalho (2011) sobre a necessidade de aprimoramento das dimensões do instrumento, estudos voltados para refinamento e ampliação da representatividade de construtos das dimensões e acúmulo dos achados referentes às evidências psicométricas estão sendo realizados. Exemplo disso são as pesquisas conduzidas por Carvalho, Souza e Primi (2014c), cujo foco foi a dimensão Conscienciosidade, e Carvalho, Sette, Capitão e Primi (2014b), com foco na dimensão Necessidade de Atenção. Ressalta-se que buscou-se replicar nesta pesquisa, especificamente voltada para a dimensão Isolamento, os procedimentos utilizados nos estudos supracitados.

De acordo com Carvalho e Primi (in press), a dimensão Isolamento do IDCP é composta por 11 itens $(\alpha=0,85)$ refere-se principalmente à preferência por ficar sozinho com evitação ao convívio social e diminuição no prazer com relacionamentos. A título de exemplo, "Interesso-me pouco em fazer amizades" concerne como um dos itens dessa dimensão. Ainda de acordo com esses autores, o transtorno da personalidade mais representado pelos itens desse fator é o esquizoide. Segundo Millon (2011), o funcionamento central esquizoide diz respeito a uma ausência de prazer nos relacionamentos interpessoais, com demarcada preferência por ficar sozinho. Pessoas com esse funcionamento são geralmente percebidas pelos outros como desapegadas emocionalmente, distantes, introvertidas, despreocupadas e indiferentes. Apesar da estreita relação da dimensão Isolamento com o funcionamento esquizoide, outros funcionamentos também apresentam características que se relacionam com os elementos avaliados pela dimensão. Exemplo disso é o funcionamento esquizotípico e o funcionamento evitativo, caracterizados pela excentricidade nos pensamentos e comportamentos e pela ansiedade exagerada em situações sociais, respectivamente (APA, 2003, 2013; Millon, 2011).

Do ponto de vista empírico, Abela (2013) realizou um levantamento do perfil de características patológicas da personalidade, com base no IDCP, de pacientes diagnosticados com transtor- 
nos da personalidade. Entre outros resultados, observou-se que a dimensão Isolamento obteve a pontuação mais elevada entre os pacientes com transtorno da personalidade esquizoide. Também os sujeitos diagnosticados com transtorno da personalidade esquizotípico e evitativo apresentaram pontuações altas nessa dimensão, ainda que de maneira menos acentuada.

Diante dos dados aqui apresentados, verifica-se que a dimensão foco deste estudo, Isolamento, está relacionada mais intimamente a alguns funcionamentos patológicos da personalidade. Apesar disso, configura-se como um grupo coeso de itens avaliando um construto latente comum. Essa dimensão apresentou em estudos anteriores adequação em suas propriedades psicométricas, mas a continuidade dos estudos de validade é necessária para o acúmulo de evidências, além da possibilidade de refinamento, atualização com a literatura e ampliação da representatividade de construto dessa dimensão. Nesse sentido, replicando os procedimentos de pesquisas anteriores, o presente estudo tem como objetivo revisar a dimensão Isolamento do IDCP. Para além da revisão e verificação das propriedades psicométricas da dimensão, buscou-se também encontrar fatores compondo essa dimensão, de modo que possam ser futuramente utilizados na investigação de perfis específicos de acordo com o construto latente avaliado.

\section{Método}

$\mathrm{O}$ método foi dividido em duas etapas. A primeira etapa para a revisão da dimensão isolamento e a segunda etapa para a verificação das propriedades psicométricas da dimensão Isolamento revisada. Tal qual mencionado, os procedimentos utilizados foram baseados em Carvalho et al. (2014b) e Carvalho et al. (2014c).

\section{Etapa I - Revisão da Dimensão Isolamento}

A primeira etapa foi realizada em seis fases, sendo que na primeira fase foi realizado o levantamento bibliográfico dos construtos referentes à dimensão Isolamento, tendo como principal base para o levantamento, características típi- cas do transtorno da personalidade esquizoide. As fontes utilizadas inicialmente foram a seção 3 do DSM-5 (APA, 2013), as sentenças e definições das facetas do Personality Inventory for DSM-5 (PID-5; Krueger, Derringer, Markon, Watson, \& Skodol, 2011), as definições das dimensões do Shedler-Westen Assessment Procedure (SWAP-200; Shedler \& Westen, 2004) e as sentenças presentes no modelo de Clark (1990) que embasa o Schedule for Nonadaptive Personality (SNAP). Contudo, a seção 3 do DSM-5 foi abandonada por não tratar das características tipicamente relacionadas à dimensão Isolamento do IDCP. Ainda, ressalta-se que a literatura acerca da teoria de Millon não foi utilizada na revisão já que na versão original do IDCP (Carvalho $\&$ Primi, in press) foi largamente considerada.

A partir disso, selecionou-se os seguintes referenciais, Personality Inventory for DSM 5 (PID-5; Krueger et al., 2011); as dimensões avaliadas pelo Shedler-Westen Assessment Procedure (SWAP; Westen \& Shedler, 1999); e as dimensões elencadas por Anna Clark (1990), que dão base para o Schedule for Nonadaptive Personality (SNAP).

$\mathrm{Na}$ segunda fase foram realizadas as traduções dos construtos componentes às referências utilizadas, organizadas em planilha eletrônica e traduzidas de maneira independente pelos autores da pesquisa. As traduções foram comparadas e chegou-se a um consenso, gerando uma tabela final em português. Os construtos foram operacionalizados na terceira fase, ou seja, foram criados novos itens com base nos construtos selecionados. Na quarta fase, os autores selecionaram os itens mais adequados, de acordo com a redação dos itens, para compor a dimensão revisada. Procurou-se não selecionar um número amplo de itens representando uma mesma característica, buscando diminuir a redundância entre os mesmos.

$\mathrm{Na}$ fase seguinte (quinta), os itens selecionados foram agrupados de acordo com seu conteúdo em categorias arbitrárias desenvolvidas pelos autores, facilitando a análise da representatividade de conteúdo dos itens. Além disso, buscou-se alocar os itens da versão original da dimensão Isolamento nas mesmas categorias. 
Essa categorização objetivou diminuir o número de itens selecionados até este momento, facilitando a visualização de itens redundantes e/ou categorias relativamente infladas quando comparadas às demais. Na sexta e última fase, foram eliminados em consenso alguns dos novos itens, mantendo-se os itens considerados pelos autores como mais adequados, tanto em termos de conteúdo (representatividade do construto) quanto em termos de redação.

\section{Etapa II - Verificação das Propriedades Psicométricas da Dimensão Isolamento Revisada}

Participantes. Foram participantes da pesquisa 213 sujeitos, com idades variando entre 18 e 69 anos $(M=25,56 ; D P=8,70)$, sendo a maioria do sexo feminino ( $N=159 ; 74,3 \%)$, com escolaridade referindo-se ao ensino superior incompleto $(83,6 \%)$, seguido por ensino superior completo $(8,9 \%)$, acessados por conveniência em duas universidades particulares do interior do Estado de São Paulo, em salas de aula e também nos campi dessas universidades. Além disso, 7,5\% dos sujeitos responderam que já fizeram tratamento psiquiátrico e $4,7 \%$ fazem, e $5,1 \%$ já fizeram uso de medicamento psicotrópico. Em relação a acompanhamento psicológico, 23,4\% dos participantes relataram ter passado por acompanhamento psicoterapêutico e apenas 7,5\% ainda fazem psicoterapia.

Instrumentos. O Inventário Dimensional Clínico da Personalidade (IDCP; Carvalho \& Primi, in press), foi utilizado nesta pesquisa em sua versão revisada composta por 191 itens agrupados em 12 dimensões sendo elas Conscienciosidade, Instabilidade de Humor, Isolamento, Dependência, Necessidade de Atenção, Autossacrifício, Impulsividade, Evitação a Críticas, Grandiosidade, Desconfiança, Excentricidade e Agressividade. Vale ressaltar que foi aplicada a versão revisada da dimensão Isolamento. Os itens são respondidos em escala tipo Likert de quatro pontos, com as respostas variando entre "não tem nada a ver comigo" (1) e "tem muito a ver comigo" (4). O IDCP apresentou evidências de validade e de fidedignidade adequadas (Carvalho, 2011). O tempo aproximado de aplicação foi de aproximadamente 25 minutos.
A versão brasileira do Inventário NEO de Personalidade Revisado (NEO-PI-R; Costa \& McCrae, 2009) também foi aplicada, sendo consideradas apenas as dimensões Neuroticismo e Extroversão, selecionadas por estarem relacionadas a um funcionamento mais patológico (depressividade, ansiedade, vulnerabilidade e oscilação no humor) e à tendência a se expressar com as pessoas, falar em público, ser comunicativo e enérgico, respectivamente. $\mathrm{O}$ NEO-PI-R é um teste de autorrelato, composto por 240 itens reunidos em cinco dimensões e em suas respectivas facetas, que são respondidos em uma escala tipo Likert de 1 a 5 pontos variando entre "discordo fortemente" (1) e "concordo fortemente" (5). As propriedades psicométricas da versão brasileira do NEO-PI-R foram consideradas adequadas (Costa \& McCrae, 2009). O tempo aproximado de aplicação deste instrumento é de 20 minutos.

Também foi utilizado o Personality Inventory for DSM-5 (PID-5; Krueger et al., 2011). O instrumento é um inventário de autorrelato para avaliação de características patológicas da personalidade composto por 220 itens representando 25 facetas (agrupadas em cinco dimensões), que devem ser respondidos em uma escala tipo Likert de 4 pontos (sendo zero igual a "muito falso ou frequentemente falso" e três igual "muito verdadeiro ou frequentemente verdadeiro"). Não foram encontrados estudos nacionais verificando as propriedades psicométricas da versão brasileira do instrumento, mas Krueger et al. (2011) apresentam dados que indicam a adequação da versão original do teste. Para este estudo foram consideradas as facetas Ansiedade, Depressividade, Evitação de Intimidade e Evitação.

Procedimentos. O projeto que embasou este estudo foi submetido a um Comitê de Ética em Pesquisa e após ser aprovado (C.A.A.E. 21992113.1.0000.5514) e autorizado, iniciou-se a coleta. A aplicação foi realizada de forma coletiva dentro de salas de aula, em uma única sessão para cada turma, sendo necessário, em média, 50 minutos, bem como de forma individual, nos casos dos participantes que se encontravam no campus da universidade. Primeiro foram explicados os objetivos da pesquisa e após os sujeitos 
consentirem em participar do estudo os mesmos assinaram o Termo de Consentimento Livre e Esclarecido e em seguida responderam aos instrumentos da pesquisa.

Os dados foram, então, tabulados e realizadas as análises estatísticas. Em um primeiro momento o número de fatores a serem mantidos na análise fatorial exploratória foi verificado com base na análise paralela para variáveis policóricas (Hayton, Allen, \& Scarpello, 2004; Watkins, 2006), por meio do software R versão 2.15.3.

Após esse procedimento, foi gerado um banco de dados para o software MPlus versão 6.12 com o intuito de realizar o procedimento analítico exploratory structural equation modeling (E-SEM) visando a análise fatorial exploratória com índices de ajuste ao modelo. O método de extração utilizado, Maximum Likelihood Robust (MLR), que tem como aspecto nuclear ser robusto para desvios de normalidade, sendo também adequado para análises com variáveis policóricas. Ressalta-se, ainda, que o ajustamento nos índices confirmatórios também sugere a adequação da amostra para a realização da análise. Por fim, os fatores encontrados compondo a dimensão revisada Isolamento foram correlacionados com as duas dimensões do NEO-PI-R e com as facetas do PID-5.

Resultados. Na primeira fase, foram utilizadas as bases já relatadas, quais sejam, o PID5, o SWAP e as dimensões proposta por Clark (1990), devido a importância e destaque que as mesmas tem no cenário atual em diagnósticos em transtornos da personalidade, além de apresentarem características relacionadas ao conteúdo avaliado pela dimensão Isolamento. Já na segunda fase, foram definidos quais construtos seriam utilizados dos modelos citados, que mais se relacionam com a dimensão Isolamento. No que se refere ao PID-5, foram utilizadas as facetas Evitação de Intimidade que trata de comportamentos evitativos à proximidade ou intimidade nos relacionamentos e evitação de relacionamentos afetivos e sexuais; Evitação, que diz respeito à evitação de situações sociais, contato e atividades em contexto social; Ansiedade, que engloba itens tratando de uma preocupação generaliza- da; e, Depressividade, referente ao humor triste, sentimento de desvalia e pouca perspectiva positiva em relação ao futuro. Em relação ao SWAP, selecionou-se somente à dimensão Orientação Esquizoide, que demonstra predominantemente a característica de indiferença em relação às pessoas, dificuldades de comunicação, incapacidade de compreensão de ideias abstratas e ausência de empatia em suas relações. E, em relação à proposta da Clark (1990), duas facetas foram selecionadas. A faceta Frieza Emocional, na qual são observadas características de frieza emocional explícita, baixa capacidade de empatia, ausência de demonstração de emoções intensas, assim como afeto, poucos gestos e expressões faciais de reciprocidade e pouca capacidade responsiva; e, Isolamento Social, na qual o sujeito faz escolhas por atividades solitárias evitando as de convívio, isolamento social e ausência de amigos próximos.

Na sequência (terceira fase), foram desenvolvidos novos itens baseados nos modelos já citados. Foi desenvolvido um total de 145 itens distribuídos de acordo com as facetas e seus construtos dos modelos citados anteriormente. Então, na quarta fase, dos 145 itens desenvolvidos com base nos cinco construtos selecionados, 55 foram pré-selecionados em consenso pelos autores do estudo, tendo como critério a seleção dos itens melhor redigidos e que mais se adequaram ao conteúdo tipicamente relacionado à dimensão Isolamento. $\mathrm{Na}$ Tabela 1 está apresentada a distribuição de itens nos construtos, considerando os itens desenvolvidos e os pré-selecionados.

$\mathrm{Na}$ quinta fase, os itens foram agrupados em categorias definidas pelos autores, com o intuito de verificar a representatividade dos novos itens elaborados, bem como investigar a representatividade dos 11 itens originais da dimensão Isolamento. Junto a isso, na última fase, o número de itens pré-selecionados foi reduzido, chegando a um total de 28 itens (novos) e 11 originais. Nessa seleção final foram excluídos itens redundantes, que sobrecarregaram as categorias criadas na fase anterior. Os dados dessas duas últimas fases podem ser observados na Tabela 2. 
Tabela 1

Conjunto de Itens Selecionados em Consenso

\begin{tabular}{lccc}
\hline & \multicolumn{3}{c}{ Itens } \\
\cline { 2 - 4 } Construtos & Criados & Pré-selecioandos & Originais \\
\hline Evitação de Intimidade & 22 & 11 & 1 \\
Evitação & 48 & 11 & 2 \\
Isolamento Social & 17 & 16 & 4 \\
Frieza Emocional & 33 & 9 & 1 \\
Orientação Esquizoide & 25 & 8 & 3 \\
Não Categorizados & & & 11 \\
\hline Total & 145 & 55 & \\
\hline
\end{tabular}

Tabela 2

Conjunto de Itens Selecionados em Consenso

\begin{tabular}{lccc}
\hline Categorias & Pré-selecionados & Selecionados & Originais \\
\hline Evitação de Intimidade & 11 & 6 & 1 \\
Desinteresse social & 11 & 6 & 2 \\
Isolamento social & 16 & 6 & 4 \\
Frieza Emocional & 9 & 5 & 1 \\
Dificuldade de Comunicação & 8 & 5 & -- \\
Não Categorizados & -- & -- & 3 \\
\hline Total & 55 & 28 & 11 \\
\hline
\end{tabular}

Na Tabela 2, no que se refere às categorias, Evitação de Intimidade caracteriza-se por atitudes de afastamento social e evitação de contatos mais íntimos, assim como de falar de si para outros; Desinteresse social agrupa itens que demonstram a evitação de formação de amizades e a ausência ou quase ausência de interesse por sentimentos dos outros ou pelas pessoas; Isolamento Social contém itens que evidenciam o desejo de estar só e de realizar suas atividades sempre individualmente; Frieza Emocional refere-se a características de não expressão de emoções assim como o contato com as próprias emoções; e, Dificuldade de Comunicação reúne sentenças que apresentam a ausência de empatia, a não identificação das emoções do outro e da falta de capacidade de manter a atenção do outro. Deve-se observar que na categoria Difi- culdade de Comunicação, nenhum item original se adequou e, além disso, restaram três itens originais que não se enquadraram em nenhuma das categorias criadas. Como resultado final da primeira etapa deste estudo, obteve-se 28 novos itens e o acréscimo de 11 itens originais, resultando em 39 itens para a versão final de aplicação da dimensão Isolamento do IDCP.

Na sequência as análises com base nos dados empíricos foram procedidas, sendo que a análise paralela para variáveis policóricas indicou até quatro fatores com eigenvalue expressivos não evidenciados ao acaso. A partir disso, procedeu-se à E-SEM, forçando-se soluções de um a quatro fatores, utilizando a rotação oblíqua geomin, e método de extração Maximum Likelihood Robust (MLR), considerado como um método robusto adequado para variáveis 
policóricas. Inicialmente foram analisados os índices de ajuste gerados para os quatro modelos, de modo que o mais adequado foi o modelo composto por quatro fatores, sendo, $\mathrm{X}^{2} / g l=2,11$; RMSEA $=0,075 ; \quad$ CFI $=0,747 ; \quad$ e, SMR $=0,057$. Considerando-se os pontos de corte (Hooper, Coughlan, \& Mullen, 2008), o SMR foi bom e o $\mathrm{X}^{2} / g l$ marginalmente bom, o RMSEA foi aceitável e o CFI não foi adequado.
Levando em conta os dados observados, optou-se pela manutenção da estrutura de quatro fatores. As cargas fatoriais encontradas, o número de itens mantidos por fator e os índices de consistência interna (alfa de Cronbach) podem ser visualizados na Tabela 3. Ainda, os itens que permaneceram em cada um dos fatores estão em negrito e marcados em cinza.

Tabela 3

Análise Fatorial Exploratória e Índices de Consistência Interna

\begin{tabular}{ccccc}
\hline Item & Individualismo & Isolamento Social & Evitação de Intimidade & Apatia Emocional \\
\hline 58 & $\mathbf{0 . 5 9 6}$ & 0.292 & 0.212 & 0.209 \\
77 & $\mathbf{0 . 5 7 8}$ & 0.491 & 0.360 & 0.264 \\
99 & $\mathbf{0 . 4 7 0}$ & 0.298 & -0.003 & 0.095 \\
111 & $\mathbf{0 . 7 2 8}$ & 0.331 & 0.298 & 0.295 \\
133 & $\mathbf{0 . 7 4 5}$ & 0.350 & 0.498 & 0.247 \\
207 & $\mathbf{0 . 4 9 8}$ & 0.301 & 0.022 & 0.104 \\
437 & 0.364 & 0.408 & $\mathbf{0 . 5 8 3}$ & 0.266 \\
438 & 0.173 & 0.318 & $\mathbf{0 . 7 0 4}$ & 0.184 \\
439 & 0.362 & 0.533 & $\mathbf{0 . 7 6 1}$ & 0.355 \\
441 & 0.216 & 0.508 & $\mathbf{0 . 6 4 1}$ & 0.233 \\
444 & 0.359 & $\mathbf{0 . 7 5 3}$ & 0.366 & 0.426 \\
445 & 0.304 & $\mathbf{0 . 6 2 8}$ & 0.221 & 0.404 \\
446 & 0.222 & $\mathbf{0 . 7 6 9}$ & 0.239 & 0.437 \\
449 & 0.196 & $\mathbf{0 . 7 5 1}$ & 0.319 & 0.392 \\
456 & 0.136 & 0.630 & 0.301 & $\mathbf{0 . 7 9 3}$ \\
457 & 0.137 & 0.328 & 0.315 & $\mathbf{0 . 6 1 6}$ \\
458 & 0.258 & 0.597 & 0.292 & $\mathbf{0 . 8 7 8}$ \\
459 & 0.226 & 0.525 & 0.334 & $\mathbf{0 . 8 6 2}$ \\
Número do item & 6 & 4 & 4 & 4 \\
$\alpha$ & 0.79 & 0.81 & 0.78 & 0.87 \\
\hline
\end{tabular}

Nota. Estão na tabela somente os itens mantidos, tornando os dados visualmente mais claros. Os itens mantidos por fator estão em negrito e as células marcadas em cinza.

De acordo com o que está apresentado na tabela, um total de 18 itens foi selecionado para a versão final da nova dimensão Isolamento. Ressalta-se aqui que explicitamente buscou-se manter um número mínimo de itens por fator encontrado, de modo a não impossibilitar o uso do instrumento pelos profissionais por conta de sua extensão. Por isso, alguns itens que apresentaram carga fatorial adequada nos fatores, não permaneceram. Basicamente, foram utilizados quatro critérios para a exclusão dos itens, sendo eles, (a) o item prejudicar ou não favorecer 
a consistência interna do fator, (b) pouca consistência interpretativa para manutenção do item no fator, (c) cargas expressivas em outros fatores (diferença menor que 0,50 nas cargas intrafatores) e (d) redundância de conteúdo entre itens em um mesmo fator. Com base nesses critérios, e no critério geral dizendo respeito à manutenção do menor número possível de itens, chegou-se ao conjunto final de itens para a versão revisada da dimensão.

Ainda, aponta-se que entre os itens excluídos, alguns poderiam ser mantidos do ponto de vista psicométrico e mesmo de conteúdo; entre- tanto, considerando o número de dimensões do instrumento, um número excessivo de itens para o IDCP tornaria inviável sua aplicação. Em relação à consistência interna dos fatores encontrados, verifica-se uma variação entre 0,78 e 0,87 e índice de 0,88 para o conjunto total de 18 itens. Após a definição da estrutura interna da dimensão, os fatores e escore total foram correlacionados entre si e com os demais testes aplicados. $\mathrm{Na}$ Tabela 4 os resultados das correlações entre os fatores e escore total da nova dimensão Isolamento e com as duas dimensões do NEO-PI-R são apresentados.

Tabela 4

Correlações entre o Escore Total, Fatores e Dimensões do NEO-PI-Rp

\begin{tabular}{|c|c|c|c|c|c|c|c|}
\hline & 1 & 2 & 3 & 4 & 5 & 6 & 7 \\
\hline 1. Individualismo & 1 & & & & & & \\
\hline 2. Isolamento Social & $0,45^{* *}$ & 1 & & & & & \\
\hline 3. Evitação de Intimidade & $0,41^{* *}$ & $0,48^{* *}$ & 1 & & & & \\
\hline 4. Apatia Emocional & $0,32^{* *}$ & $0,58^{* *}$ & $0,38^{* *}$ & 1 & & & \\
\hline 5. Escore Total & $0,81^{* *}$ & $0,76^{* *}$ & $0,73^{* *}$ & $0,71^{* *}$ & 1 & & \\
\hline 6. Neuroticismo & $0,33^{* *}$ & $0,25^{* *}$ & 0,06 & $0,14^{*}$ & $0,27^{* *}$ & 1 & \\
\hline 7. Extroversão & $-0,20^{* *}$ & $-0,29^{* *}$ & $-0,24^{* *}$ & $-0,31^{* *}$ & $-0,34^{* *}$ & $-0,08$ & 1 \\
\hline
\end{tabular}

*Significativo ao nível de $p=0,05 ; * *$ significativo ao nível de $p=0,001$.

Nota-se que as magnitudes de correlação do escore total em relação aos fatores foram sempre altas. Da mesma forma observa-se que as magnitudes entre os fatores da dimensão revisada variaram entre 0,32 e 0,58 . O escore total apresentou uma correlação baixa e positiva com Neuroticismo e baixa e negativa com Extroversão. A dimensão Neuroticismo apresentou correlações positivas com os fatores da dimensão do IDCP, sendo as mais altas com Individualismo $(r=0,33)$ e com Isolamento Social $(r=0,25)$. Diferente, Extroversão apresentou somente relações negativas, as mais altas com os fatores Isolamento Social $(r=-0,29)$ e Apatia Emocional $(r=-0,31)$. Na continuidade, estão apresentadas na Tabela 5 as correlações da dimensão revisada com as facetas de Neuroticismo.

$\mathrm{O}$ fator Individualismo apresentou maiores correlações com Embaraço/Constrangimento e
Vulnerabilidade; Isolamento Social com a faceta Depressão e Embaraço/Constrangimento; Evitação de Intimidade apresentou correlações baixas de um modo geral; e Apatia Emocional correlacionou-se mais intensamente com a faceta Depressão. O escore total apresentou correlação mais alta com Vulnerabilidade e Embaraço/ Constrangimento. Estão apresentados dados similares a estes na Tabela 6 , mas relativos à dimensão Extroversão.

De acordo com a Tabela 6 todas as correlações significativas foram também negativas. Os fatores Individualismo, Isolamento Social e Evitação de Intimidade, assim como o escore total, apresentaram correlações mais expressivas com Acolhimento e Gregarismo; e, Apatia Emocional com Acolhimento e Atividade. Na Tabela 7 estão apresentas as correlações dos fatores do IDCP com as facetas do PID-5. 
Tabela 5

Correlações entre Isolamento e Facetas de Neuroticismo

\begin{tabular}{lcccccc}
\hline & $\mathrm{A}$ & $\mathrm{R} / \mathrm{H}$ & $\mathrm{D}$ & $\mathrm{E} / \mathrm{C}$ & $\mathrm{I}$ & $\mathrm{V}$ \\
\hline Individualismo & $0,22^{* *}$ & $0,19^{* *}$ & 0,12 & $0,33^{* *}$ & $0,24^{* *}$ & $0,33^{* *}$ \\
Isolamento Social & 0,87 & $0,16^{*}$ & $0,28^{* *}$ & $0,24^{* *}$ & 0,25 & $0,23^{* *}$ \\
Evitação de Intimidade & $-0,09$ & 0,23 & 0,53 & 0,11 & $-0,12$ & 0,96 \\
Apatia Emocional & 0,14 & 0,38 & $0,24^{* *}$ & $0,16^{*}$ & $-0,74$ & $0,18^{* *}$ \\
Escore Total & 0,10 & $0,14^{*}$ & $0,19^{* *}$ & $0,29^{* *}$ & 0,94 & $0,31^{* *}$ \\
\hline
\end{tabular}

Nota. $\mathrm{A}=$ Ansiedade; $\mathrm{R} / \mathrm{H}=$ Raiva/Hostilidade; $\mathrm{D}=$ Depressão; $\mathrm{E} / \mathrm{C}=$ Embaraço/ Constrangimento; $\mathrm{I}=$ Impulsividade; $\mathrm{V}=$ Vulnerabilidade.

*Significativo ao nível de $p=0,05 ; * *$ significativo ao nível de $p=0,01$.

Tabela 6

Correlações entre Isolamento e Facetas de Extroversão

\begin{tabular}{|c|c|c|c|c|c|c|}
\hline & A & G & As & At & $\mathrm{B}$ de $\mathrm{S}$ & E P \\
\hline Individualismo & $-0,26^{* *}$ & $-0,23^{* *}$ & $-0,01$ & $-0,13^{*}$ & $-0,10$ & $-0,07$ \\
\hline Isolamento Social & $-0,26^{* *}$ & $-0,26^{* *}$ & $-0,07$ & $-0,12$ & $-0,19^{* *}$ & $-0,17^{*}$ \\
\hline Evitação de Intimidade & $-0,27^{* *}$ & $-0,28^{* *}$ & 0,01 & 0,02 & $-0,21^{* *}$ & $-0,15^{*}$ \\
\hline Apatia Emocional & $-0,23^{* *}$ & $-0,20^{* *}$ & $-0,86$ & $-0,21^{* *}$ & $-0,20^{* *}$ & $-0,21^{* *}$ \\
\hline Escore Total & $-0,34^{* *}$ & $-0,31^{* *}$ & $-0,64$ & $-0,17^{*}$ & $-0,22^{* *}$ & $-0,18^{* *}$ \\
\hline
\end{tabular}

Nota $. \mathrm{A}=$ Acolhimento $; \mathrm{G}=$ Gregarismo; $\mathrm{As}=$ Assertividade; $\mathrm{At}=$ Atividade $; \mathrm{B}$ de $\mathrm{S}=$ Busca de Sensações $; \mathrm{E} \mathrm{P}=$ Emoções Positivas.

*Significativo ao nível de $p=0,05 ; *$ significativo ao nível de $p=0,01$.

Tabela 7

Correlações entre Isolamento e Facetas do PID-5

\begin{tabular}{|c|c|c|c|c|}
\hline & Ansiedade & Depressividade & Evitação de Intimidade & Evitação \\
\hline Individualismo & $0,40^{* *}$ & $0,40^{* *}$ & $0,21^{* *}$ & $0,48^{* *}$ \\
\hline Isolamento Social & $0,28^{* *}$ & $0,40^{* *}$ & $0,20^{* *}$ & $0,59^{* *}$ \\
\hline Evitação de Intimidade & $0,29^{* *}$ & $0,18^{* *}$ & $0,25^{* *}$ & $0,44^{* *}$ \\
\hline Apatia Emocional & $0,23^{* *}$ & $0,44^{* *}$ & $0,17^{*}$ & $0,60^{* *}$ \\
\hline Escore Total & $0,40^{* *}$ & $0,47^{* *}$ & $0,28^{* *}$ & $0,69^{* *}$ \\
\hline
\end{tabular}

*Significativo ao nível de $p=0,05 ; * *$ significativo ao nível de $p=0,01$.

No que tange as relações encontradas com o escore total, verifica-se que a maior relação foi com a faceta Evitação e a menor com Evitação de Intimidade, o mesmo ocorreu com todos os fatores da dimensão do IDCP em relação a essas duas facetas do PID-5. Individualismo também apresentou magnitudes de correlação mais evidentes com Ansiedade e Depressividade; Isolamento Social também com Depressividade;
Evitação de Intimidade (do IDCP) apresentou as correlações mais baixas; e Apatia Emocional também com Depressividade.

\section{Discussão}

Buscou-se obter um novo conjunto de itens para a dimensão Isolamento do IDCP, com propriedades psicométricas satisfató- 
rias no que se refere a evidências de validade com base na estrutura interna e relações com variáveis externas, bem como aos índices de fidedignidade. Na revisão, foram considerados os itens originais do IDCP (Carvalho \& Primi, in press), e procurou-se desenvolver prioritariamente itens avaliando a faixa patológica da personalidade, especificamente, dos aspectos relacionados ao construto latente à dimensão Isolamento.

Essa dimensão, tal qual já relatado, envolve características como a preferência por ficar sozinho, acompanhada pela diminuição no prazer em relacionamentos e evitação de situações de sociais, e está relacionada às características típicas do transtorno da personalidade esquizoide (Millon, 2011). Foi elaborado neste estudo um conjunto de 28 itens que foram aplicados juntamente aos 11 itens originais da dimensão.

$\mathrm{O}$ agrupamento dos itens (originais e novos) da dimensão Isolamento em categorias facilitou a análise da cobertura a que o conjunto de itens se refere, sugerindo que a literatura acessada como base está sendo abarcada. As categorias Evitação de Intimidade e Evitação Social possuem elementos propostos no PID-5 (Krueger et al., 2011), assim como elementos evidenciados no SWAP (Westen \& Shedler, 1999) que foram reelaborados na categoria Dificuldade de Comunicação, e nas propostas de Clark (1990) para o desenvolvimento dos itens presentes nas categorias Isolamento Social e Frieza Emocional. Dos itens originais, três não se adequaram a nenhuma das categorias e na categoria Dificuldade de Comunicação nenhum item original do IDCP se adequou.

A partir da investigação das propriedades psicométricas do conjunto de itens novos e originais, estabeleceu-se uma solução composta por quatro fatores. Deve-se considerar que alguns dos índices de ajuste utilizados demonstraram adequação, mas especificamente o CFI não atingiu os pontos de corte desejados, o que deve ser levado em consideração, sobretudo, em futuros estudos buscando replicar a estrutura aqui estabelecida. Após refinamento, isto é, a exclusão de itens de acordo com os critérios já apresentados, a nova dimensão Isolamento ficou composta por 18 itens, sendo seis originais (todos presentes no primeiro fator encontrado) e 12 novos (distribuídos nos três últimos fatores da dimensão Isolamento). O primeiro fator, Individualismo ( 6 itens) caracteriza-se por uma preferência do sujeito em realizar atividades sozinho, podendo envolver irritação quando colocado em situações que envolvem contato com as pessoas (exemplo de item: Prefiro fazer as coisas sozinho.). O fator Isolamento Social (4 itens) agrupa itens sobre uma tendência a preferir não ter contato com as pessoas (Exemplo de item: Não entendo porque as pessoas gostam tanto de fazer amizades.). No fator três, Evitação de Intimidade (4 itens), estão presentes itens que denotam uma clara preferência por não estabelecer relações íntimas e não compartilhar informações de si com os outros (exemplo de item: Dificilmente permito que alguém tenha intimidade comigo.). E o último fator, Apatia Emocional (4 itens), diz respeito à dificuldade para se entusiasmar com as situações do cotidiano (exemplo de item: Não entendo como as pessoas ficam tão empolgadas com as coisas.). As características compondo os fatores encontrados estão reportadas na literatura de base para o desenvolvimento da dimensão revisada (Clark, 1990; Krueger et al., 2011; Millon, 2011; Shedler \& Westen, 2004).

Os dados encontrados no que se refere à solução fatorial da versão revisada da dimensão Isolamento conferem evidência de validade com base na estrutura interna para essa dimensão, já que os fatores encontrados apresentaram interpretabilidade e coerência em relação à literatura de base utilizada neste estudo. Ao lado disso, também foram observados índices adequados no que diz respeito à consistência interna da dimensão revisada e os fatores que a compõem, sobretudo, considerando o número reduzido de itens (Nunnally, 1978). 
Os 18 itens componentes da versão final revisada da dimensão Isolamento foram correlacionados entre si e com as demais facetas e dimensões do NEO-PI-R e PID-5. As correlações entre os fatores de Isolamento e o escore total indicam, por um lado, a presença de um construto latente comum subjacente à dimensão. O que é também corroborado pelos índices observados de consistência interna. Contudo, por outro lado, considerando as magnitudes de correlação evidenciadas, isto é, de baixas a moderadas, verifica-se também a possibilidade da obtenção de diferentes perfis de respondentes à dimensão Isolamento. Por exemplo, duas pessoas podem apresentar uma pontuação alta no escore total dessa dimensão, mas também obter um perfil distinto nos fatores que a compõem. Mais que esperado, a obtenção de fatores coesos e relacionados, mas ainda assim distintos, era desejável para a dimensão revisada, permitindo um maior refinamento quanto à avaliação feita sobre um indivíduo.

Ao lado disso, as correlações positivas dos fatores e escore total de Isolamento com Neuroticismo indicam uma tendência dos itens dessa dimensão para a faixa patológica, considerando que essa dimensão do NEO-PI-R avalia aspectos mais disfuncionais da personalidade (Costa \& McCrae, 2009). Os fatores de Isolamento que apresentaram maiores magnitudes de correlação com essa dimensão foram aqueles mais tipicamente relacionados com a preferência por ficar sozinho (Individualismo e Isolamento Social), o que deve ser investigado em estudos futuros. No que concerne à dimensão Extroversão, todas as relações foram negativas, o que também era esperado, pois pontuações altas nessa dimensão do NEO-PI-R indicam uma preferência por estar entre as pessoas (Costa \& McCrae, 2009). Compreende-se como evidência de validade com base nas dimensões Neuroticismo e Extroversão do NEO-PI-R os dados encontrados, já que é esperado que a dimensão Isolamento demonstrasse uma tendência para avaliação de aspectos patológicos da personalidade, o que vale para os demais fatores do IDCP, mas também refira-se a uma preferência para ficar isolado das pessoas.

Especificamente sobre as correlações com as facetas de Neuroticismo, o fator Individualidade correlacionou-se de maneira mais expressiva com Embaraço/Constrangimento e Vulnerabilidade, indicando que as pessoas com altas pontuações nesse fator do IDCP tendem a apresentar vergonha e timidez associadas à ansiedade social e suscetibilidade a estresse e a agressões psicológicas. Também o fator Isolamento Social correlacionou-se de maneira importante com Embaraço/Constrangimento, mas também com a faceta Depressão, sugerindo que pessoas que preferem evitar o convívio social também apresentam uma tendência à timidez e a não se colocar socialmente e também ao humor triste, possivelmente pelo próprio isolamento do convívio social. Também Apatia emocional demonstrou correlação com Depressão, o que parece pertinente, já que o fator e a faceta tratam do humor das pessoas. Futuros estudos devem investigar essas relações de maneira mais detalhada. Diferentemente, Evitação de Intimidade foi o fator do IDCP que apresentou as correlações mais baixas com as facetas de Neuroticismo, sugerindo que essa dimensão do NEO-PI-R não apresenta itens avaliando características acerca da dificuldade por estabelecer vínculos íntimos com as pessoas, o que pode ser observado pelas definições das facetas dessa dimensão (Costa \& McCrae, 2009).

Também foram investigadas as relações entre Isolamento e as facetas de Extroversão. Observou-se que todas as correlações significativas foram também negativas, sugerindo novamente que a dimensão do IDCP está relacionada a uma preferência por não se relacionar com as pessoas e um rebaixamento na energia dispendida para as atividades do cotidiano, tal qual avaliado pela dimensão do NEO-PI-R (Costa \& McCrae, 2009). Grande parte das relações encontradas dos fatores de Isolamento apre- 
sentaram relação expressiva com as facetas Acolhimento e Gregarismo. Isso sugere que esses fatores (sobretudo, Individualismo, Isolamento Social e Evitação de Intimidade) estão relacionados com uma tendência a não buscar afeto, amizade e estimulação social (Costa \& McCrae, 2009). Também o fator Apatia Emocional apresentou relação com Acolhimento, o que também é teoricamente pertinente, e com Atividade, sugerindo que essa dimensão se refere a uma tendência a ausência de energia, vigor e agilidade.

Também correlações dos fatores de Isolamento com algumas facetas do PID-5 foram verificadas, nas quais notam-se maiores magnitudes com a faceta Evitação e menores com a faceta Evitação de Intimidade, ambas do PID-5. Isso sugere que a dimensão Isolamento trata de atitudes que evitam o contato social em geral e a preferência por ficar só conforme a faceta Evitação, como descritas e não tanto com a esquiva especificamente de relações amorosas e/ou sexuais, elemento esse subjacente à faceta Evitação de Intimidade (Krueger et al., 2011). Como observado similarmente nas facetas de Neuroticismo (NEO-PI-R), Individualismo e Isolamento Social demonstraram relações expressivas com a faceta Depressividade do PID-5, o que também é verdadeiro para Apatia Emocional. Ainda, Evitação de Intimidade foi o fator de Isolamento com as menores relações com o PID-5, apesar deste último apresentar uma faceta com o mesmo nome. Contudo, o fator do IDCP avalia a preferência por não estabelecer relações íntimas de modo mais geral, e não somente em relacionamentos amorosos ou sexuais, como no caso do PID-5 (Krueger et al., 2011). Diferente, o fator com o mesmo nome, do IDCP, avalia a preferência por não estabelecer relações íntimas de modo mais geral, e não somente em relacionamentos amorosos ou sexuais.

Compreende-se que as relações encontradas entre o IDCP e o PID-5 também são evidências de validade com base em variável externa para a dimensão Isolamento, já que foram teoricamente coerentes. Além disso, vale ainda ressaltar que as relações encontradas entre o IDCP e o PID-5 foram de magnitude maior em relação ao IDCP e o NEO-PI-R. Esse dado é favorável, considerando que o IDCP e o PID-5 se propõem a avaliar características patológicas da personalidade, e o NEO-PI-R foi desenvolvido para avaliar características mais típicas da população.

\section{Considerações Finais}

Entende-se que o objetivo deste estudo foi atingido, qual seja, revisar a dimensão Isolamento, de modo a manter os aspectos patológicos de seus itens, coeficientes de fidedignidade adequados, e interpretabilidade coerente das características que compõem a dimensão. Para tanto, dois estágios amplos foram utilizados, de revisão conceitual e posteriormente, de revisão empírica, da dimensão.

Este estudo configura-se como uma primeira aproximação e exploração da dimensão revisada, ainda que a mesma contemple também alguns de seus itens originais. Nesse sentido, futuros estudos devem buscar aplicar essa dimensão do IDCP com outros instrumentos, bem como em outras amostras, sobretudo, em pacientes com diagnóstico psiquiátrico.

Para além disso, algumas limitações deste estudo devem ser notadas. Primeiro, a amostra, ainda que suficiente para as análises (Tabachnick \& Fidell, 2007), não é representativa da população e também não apresenta dados sobre diagnósticos psiquiátricos. Deve-se considerar como uma limitação o não uso de juízes externos nesta etapa; por outro lado, os autores do estudo aprofundaram seus conhecimentos tanto no instrumento quanto nas características tipicamente relacionadas à dimensão Isolamento, o que é favorável para realização da fase. Além disso, neste estudo, outras dimensões do IDCP, e também do NEO-PI-R e PID-5, e suas relações com a nova dimensão Isolamento, não foram verificadas. Ainda, apesar dos dados favoráveis com a dimensão revisada, o seu uso clínico ainda não foi testado, o que deve ser realizado em pesquisas futuras. 


\section{Referências}

Abela, R. K. (2013). Evidências de validade para o Inventário Dimensional Clínico da Personalidade (IDCP) com base nos perfis de participantes com diagnostico psiquiátrico (Tese de doutorado, Universidade Federal de São Paulo, SP, Brasil).

American Psychiatric Association. (2003). Manual diagnóstico e estatístico de transtornos mentais $D S M-I V$-TR (4. ed.). Porto Alegre, RS: Artmed.

American Psychiatric Association. (2013). Diagnostic and statistical manual of mental disorders ( $5^{\text {th }}$ ed.). Arlington, VA: Author.

Carvalho, L. F. (2011). Desenvolvimento e verificação das propriedades psicométricas do Inventário Dimensional Clínico da Personalidade (Tese de doutorado, Universidade São Francisco). Recuperado em https://www.usf.edu.br/galeria/getImage/427/606165816189410.pdf

Carvalho, L. F., Bartholomeu, D., \& Silva, M. C. R. (2010). Instrumentos para avaliação dos transtornos da personalidade no Brasil. Avaliação Psicológica, 9(2), 289-298. Recuperado em http:// pepsic.bvsalud.org/pdf/avp/v9n2/v9n2a13.pdf

Carvalho, L. F., \& Primi, R. (in press). Prototype matching of personality disorders with the Dimensional Clinical Personality Inventory. Psicologia: Teoria e Pesquisa.

Carvalho, L. F., Primi, R., \& Stone, G. E. (2014a). Psychometric properties of the Inventário Dimensional Clínico da Personalidade (IDCP) using the Rating Scale Model. Avances en Psicología Latinoamericana, 32(3). doi:10.12804/ ap132.03.2014.09

Carvalho, L. F., Sette, C. P., Capitão, C. G., \& Primi, R. (2014b). Propriedades psicométricas da versão revisada da dimensão necessidade de atenção do Inventário Dimensional Clínico da Personalidade. Temas em Psicologia, 22(1), 147-160. doi:10.9788/TP2014.1-12

Carvalho, L. F., Souza, B. D. B., \& Primi, R. (2014c). Psychometric properties of the revised conscientiousness dimension of Inventário Dimensional Clínico da Personalidade (IDCP). Revista de Psiquiatria do Rio Grande do Sul, 36, 23-31. doi:10.1590/2237-6089-2013-0024

Clark, L. A. (1990). Toward a consensual set of symptom clusters for assessment of personality disorder. In J. Butcher \& C. Spielberger (Eds.), Advances in personality assessment (Vol. 8, pp. 243-266). Hillsdale, NJ: Lawrence Erlbaum.

Costa, P. T., Jr., \& McCrae, R. R. (2009). NEO-PI$-R$ - Inventário de Personalidade NEO Revisado - Manual. São Paulo, SP: Vetor.

Hayton, J. C., Allen, D. G., \& Scarpello, V. (2004). Factor retention decisions in exploratory factor analysis: A tutorial on parallel analysis. Organizational Research Methods, 7(2), 191-205. doi:10.1177/1094428104263675

Hooper, D., Coughlan, J., \& Mullen, M. R. (2008). Structural equation modelling: Guidelines for determining model Fit. The Electronic Journal of Business Research Methods, 6(8), 53-60. Retrieved from http://arrow.dit.ie/buschmanart

Kendler, K. S., Kupfer, D., Narrow, W., Phillips, K., \& Fawcett, J. (2009, October 21). Guidelines for making changes to DSM- $V$. Retrieved from http://www.dsm5.org/ProgressReports/ Documents/Guidelines-for-Making-Changes-to-DSM_1.pdf

Krueger, R. F., Derringer, J., Markon, K. E., Watson, D., \& Skodol, A. E. (2011). Initial construction of a maladaptive personality trait model and inventory for DSM-5. Psychological Medicine, 8, 1-12. doi:10.1017/S0033291711002674

Millon, T. (2011). Disorders of personality: Introducing a DSM/ICD spectrum from normal to abnormal ( $3^{\text {rd }}$ ed.). Hoboken, NJ: Wiley.

Millon, T., Grossman, S., \& Tringone, R. (2010). The Millon Personality Spectrometer: A tool for personality spectrum analyses, diagnoses, and treatments. In T. Millon, R. F. Krueger, \& E. Simonsen (Eds.), Contemporary directions in psychopathology: Scientific foundations of the DSM-V and ICD-11 (pp. 391-418). New York: Guilford.

Millon, T., Millon, C. M., Meagher, S., Grossman, S., \& Ramanath, R. (2004). Personality disorders in modern life ( $2^{\text {nd }}$ ed.). Hoboken, NJ: Wiley.

Nunnally, J. C. (1978). Psychometric theory ( $2^{\text {nd }}$ ed.). New York: McGraw-Hill.

Shedler, J., \& Westen, D. (2004). Dimensions of personality pathology: An alternative to the five factor model. American Journal of Psychiatry, 161, 1743-1754. Retrieved from http://journals.psychiatryonline.org/article. aspx? articleid $=177083$ 
Skodol, A. E., Clark, L. A., Bender, D. S., Krueger, R. F., Morey, L. C., Verheul, R., ...Oldham, J. M. (2011). Proposed changes in personality and personality disorder assessment and diagnosis for DSM-5, Part I: Description and rationale. Personality Disorders: Theory, Research, and Treatment, 2(1), 4-22. doi:10.1037/a0021891

Tabachnick, B. G., \& Fidell, L. S. (2007). Using multivariate statistics $\left(5^{\text {th }}\right.$ ed.). Boston, MA: Allyn \& Bacon.

Watkins, M. W. (2006). Determining parallel analysis criteria. Journal of Modern Applied Statistical Methods, 5(2), 344-346. Retrieved from http:// digitalcommons.wayne.edu/jmasm/vol5/iss2/8

Westen, D., \& Shedler, J. (1999). Revising and assessing Axis II, Part I: Developing a clinically and empirically valid assessment method. American Journal of Psychiatry, 156, 258-272. Retrieved from http://journals.psychiatryonline.org/article.aspx?articleid $=173271$
Widiger, T. A., \& Lowe, J. R. (2008). A dimensional model of personality disorder: Proposal for DSM-V. The Psychiatric Clinics of North America, 31(3), 363-378. doi:10.1016/j. psc.2008.03.008

Zimmerman, M. (2011). Is there adequate empirical justification for radically revising the personality disorders section for DSM 5? Personality Disorders: Theory, Research, and Treatment, 25(2), 206-221. doi:10.4088/JCP.11m06974
Recebido: 06/08/2014

$1^{a}$ revisão: 05/11/2014

$2^{a}$ revisão: 08/01/2015 Aceite final: 13/01/2015 